\title{
Phase Transition and Ordering Temperatures of TiAl-Mo Alloys Investigated by In-Situ Diffraction Experiments
}

\author{
Thomas Schmoelzer $^{1, \mathrm{a}}$, Svea Mayer ${ }^{1, \mathrm{~b}}$, F. Haupt $^{2, \mathrm{c}}$, G.A. Zickler ${ }^{3, \mathrm{~d}}$,

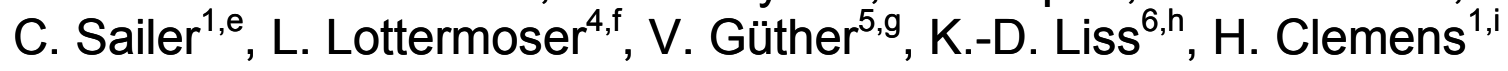 \\ ${ }^{1}$ Dept. Physical Metallurgy and Materials Testing, University of Leoben, 8700 Leoben, Austria \\ ${ }^{2} \mathrm{GfE}$ Fremat $\mathrm{GmbH}, 09599$ Freiberg, Germany \\ ${ }^{3}$ Christian Doppler Laboratory for Early Stages of Precipitation, Dept. Physical Metallurgy and \\ Materials Testing, University of Leoben, 8700 Leoben, Austria \\ ${ }^{4}$ Institute of Materials Research, GKSS Outstation in Hamburg, 22607 Hamburg, Germany \\ ${ }^{5} \mathrm{GfE}$ Metalle und Materialien $\mathrm{GmbH}, 90431$ Nuremberg, Germany \\ ${ }^{6}$ Australian Nuclear Science and Technology Organisation, NSW 2234, Menai, Australia
}

athomas.schmoelzer@unileoben.ac.at, bsvea.mayer@unileoben.ac.at, 'frank.haupt@gfe.com, dgerald.zickler@unileoben.ac.at, ${ }^{e}$ christian.sailer@stud.unileoben.ac.at, ${ }^{\prime}$ lars.lottermoser@gkss.de, gvolker.guether@gfe.com, hkdl@ansto.gov.au, 'helmut.clemens@unileoben.ac.at

Keywords: TiAl, phase diagram, diffraction, thermodynamic calculations, phase transformations

\begin{abstract}
Intermetallic TiAl alloys with a significant volume fraction of the body-centered cubic $\beta$ phase at elevated temperatures have proven to exhibit good processing characteristics during hotworking. Being a strong $\beta$ stabilizer, Mo has gained importance as an alloying element for so-called $\beta / \gamma$-TiAl alloys. Unfortunately, the effect of Mo on the appearing phases and their temperature dependence is not well known. In this work, two sections of the Ti-Al-Mo ternary phase diagram derived from experimental data are shown. These diagrams are compared with the results of in-situ high-temperature diffraction experiments using high-energy synchrotron radiation.
\end{abstract}

\section{Introduction}

During the last twenty years, research and development efforts have been undertaken to develop $\gamma$ TiAl based alloys as a replacement for Ni-based superalloys for high-temperature applications in advanced aero engines and automotive combustion engines [1]. While $\gamma$-TiAl based alloys are intended for use in high-temperature aerospace applications $\left(600-900^{\circ} \mathrm{C}\right)$, there is growing demand for TiAl alloys reasonably ductile at room temperature. One approach is to design alloys with microstructures in which homogenously distributed $\beta$ - and $\gamma$-phase are the main constituents. These alloys are termed $\beta / \gamma$-alloys [2]. Since Mo is a strong $\beta$ stabilizer, the ternary system Ti-Al-Mo is well suited for studying this type of alloys [3]. Despite the importance of Mo, the knowledge of its effect on the Ti-Al phase diagram is limited. In many TiAl alloys, three intermetallic phases are the main microstructural constituents, all of which are ordered at room temperature: $\gamma, \alpha_{2}$ and $\beta_{0}$. At elevated temperatures the ordered hexagonal $\alpha_{2}$ phase $\left(\mathrm{D}_{19}\right)$ disorders to $\alpha(\mathrm{A} 3)$ and the ordered cubic $\beta_{0}$-phase (B2) disorders to the body-centered cubic $\beta$ phase (A2) whereas the $\gamma$-phase (L1 $)$ disappears at the so-called $\alpha$-transus temperature $\left(T_{\alpha}\right)$. The phase fractions present strongly influence the mechanical properties of the material as well as the processing characteristics at hot-working temperatures. High $\beta$-phase contents, for instance, improve the deformation behavior during hotworking, but simultaneously decrease creep resistance if present at service temperatures $\left(\mathrm{T}<900^{\circ} \mathrm{C}\right)$ [4]. Two sections of the ternary phase diagram Ti-Al-Mo, based on published experimental results, are presented. To critically assess the phase diagrams, two alloys with a nominal composition of Ti45Al-3Mo (alloy A) and Ti-45Al-7Mo (alloy B) were investigated. Note that all concentrations are given in at $\%$. For determining the phase evolution and phase transition temperatures, in-situ highenergy X-ray diffraction [5] (HEXRD) experiments were conducted. The experimental results are then compared to the quasi-binary phase diagrams and the differences discussed. 


\section{Experimental Procedures}

Two sections through the Ti-Al-Mo ternary phase diagram for constant Al contents of $43 \%$ and $45 \%$ were constructed from experimental data published in the open literature [6-9]. Two model alloys were investigated which were cast by GfE Metalle und Materialien GmbH, Nuremberg, Germany, and provided in the hot-isostatically pressed condition. By means of X-ray fluorescence spectroscopy, actual compositions of alloy A and B were determined to be Ti-44.58Al-3.23Mo0.12B and Ti-43.94Al-6.78Mo-0.09B, respectively. Please note the significant difference in the Al content of the two alloys. HEXRD experiments were performed at the HARWI II beamline of the GKSS research center at the DESY synchrotron in Hamburg, Germany [5,10]. For heating the specimens, a custom built induction furnace was operated under Ar-atmosphere. The temperature of the $5 \mathrm{~mm}$ diameter and $15 \mathrm{~mm}$ long cylindrical samples was measured by a pyrometer. A mean energy of $104.7 \mathrm{keV}$ was adjusted for the $0.5 \times 0.5 \mathrm{~mm}^{2}$ beam. As detector a mar555 by MAR Research, Nordersted, Germany was employed. The samples were rapidly heated to $1000^{\circ} \mathrm{C}$, held at that temperature for $2 \mathrm{~min}$, and then heated to $1350^{\circ} \mathrm{C}$ at a rate of $2 \mathrm{~K} / \mathrm{min}$. For azimuthal integration of the acquired patterns the software fit2D was used [11]. Rietveld analysis was performed with the commercial software package TOPAS by Brucker AXS, Madison, USA. The peak areas were calculated by a self developed software tool. Individual peaks of both phases were fitted with a Gaussian function and multiplied by a weighting factor in order to obtain phase fractions by the intensity ratio method. The weighting factors were derived from the results obtained by Rietveld analysis.

\section{Results and Discussion}

Two sections of the ternary Ti-Al-Mo system are shown in Fig.1. Grey lines represent the section at $43 \% \mathrm{Al}$ whereas the phase diagram for $45 \% \mathrm{Al}$ is shown by dashed black lines. From Fig. 1 it is obvious, that the transition temperatures of the two phase diagrams differ significantly. Additionally, with increasing $\mathrm{Al}$ content, the $(\beta+\gamma)$ phase field extends to smaller Mo contents. In the case of alloy $\mathrm{B}$, this phase field is not traversed in the section for $43 \% \mathrm{Al}$.

Fig.1: Sections of the ternary Ti-Al-Mo phase diagram for Al-contents of $45 \%$ (dashed black lines) and $43 \%$ (grey lines). The compositions of the investigated alloys $\mathrm{A}$ and $\mathrm{B}$ are indicated by two vertical lines. The transition temperatures determined by in-situ HEXRD are indicated by horizontal marks and letters: a indicates the eutectoid temperature, $b$ the $\beta_{O} \rightarrow \beta$ disordering temperature, and $\mathrm{c}$ the $\alpha$-transus temperature. The letter $d$ indicates the onset of $\alpha$-phase formation upon heating of alloy B.

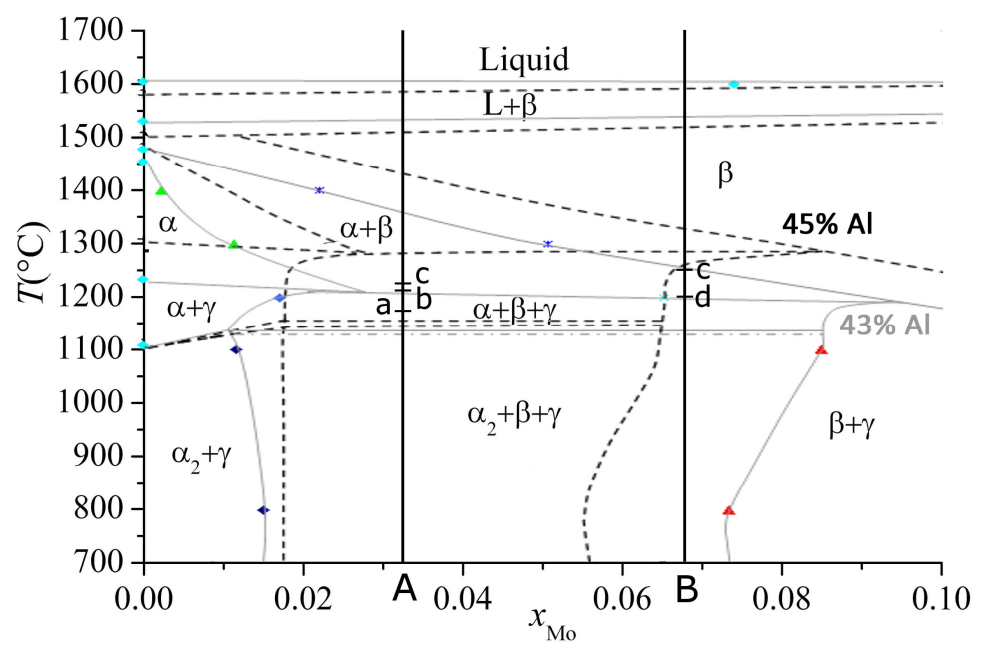

In Fig. 2 the phase evolution as determined by in-situ HEXRD experiments is presented for sample A and B. Whereas alloy A with $\sim 3 \%$ Mo shows high amounts of $\alpha / \alpha_{2}$ phase (Fig. $2 \mathrm{a}$ ), only a small fraction is observed for B with a Mo content of $\sim 7 \%$ (Fig.2b). This trend is consistent with Fig.1 predicting the $\alpha / \alpha_{2}$ phase to be stable at low temperatures only near composition A whereas for $\mathrm{B}, \alpha / \alpha_{2}$ is stable only above $1265^{\circ} \mathrm{C}$ near the composition of alloy $\mathrm{B}$. 

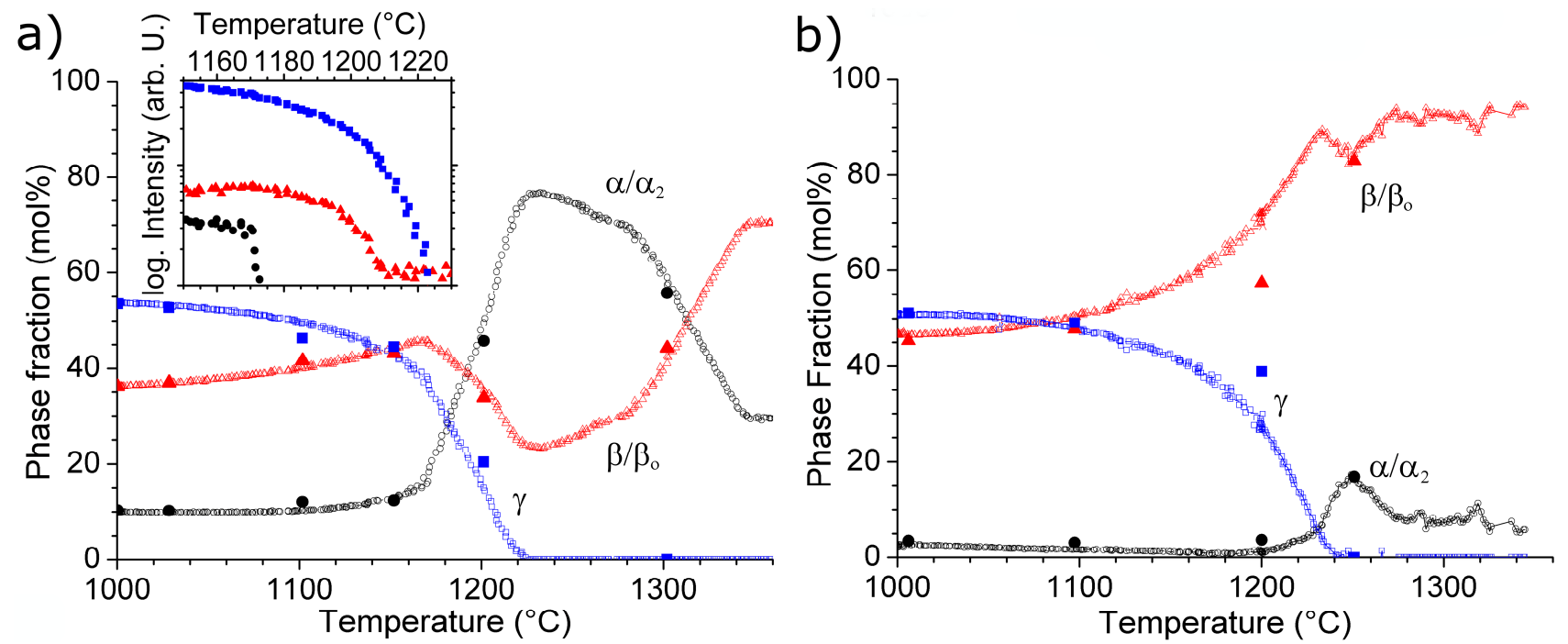

Fig.2: Phase evolution of (a) Ti-45Al-3Mo and (b) Ti-45Al-7Mo (nominal composition). Circles represent the $\alpha$-phase, whereas triangles and squares correspond to the $\beta$ - and $\gamma$-phase, respectively. Filled symbols indicate results obtained from Rietveld-analysis, open ones were gained from evaluation of peak-areas. The inset in (a) shows the $\alpha_{2} \rightarrow \alpha$ and $\beta_{\mathrm{O}} \rightarrow \beta$ transition temperature as well as $T_{\alpha}$ for alloy A i.e. $\gamma \rightarrow 0$.

The results of the Rietveld analyses are represented by the larger, filled symbols in Fig. 2. With the exception of the fits performed at $1200^{\circ} \mathrm{C}$, good accordance between the Rietveld and peak-area evaluation is observed. The deviation at $1200^{\circ} \mathrm{C}$ can be attributed to the error introduced by the proximity of the diminishing $\gamma-111$ peak to the increasing $\beta-011$ peak. Additionally, the order to disorder temperatures can be derived from the inset in Fig. 2a. Here, the intensity of the $\alpha_{2}-011$ and $\beta_{0}-001$ reflections as well as the intensity of the $\gamma-011 / 110$ double peak are displayed for alloy A. Vanishing of these peaks corresponds to the loss of order in the $\alpha_{2}$ and $\beta_{\mathrm{o}}$ phase or the dissolution of $\gamma$-phase. For alloy A these temperatures were determined to $1175^{\circ} \mathrm{C}, 1210^{\circ} \mathrm{C}$, and $1225^{\circ} \mathrm{C}$ for $\alpha_{2}, \beta_{0}$, and $\gamma$, respectively. Compared to data determined on a Ti-43Al-4Nb-1Mo alloy, the $\alpha_{2} \rightarrow \alpha$ temperature shows hardly any difference, whereas $T_{\alpha}$ is significantly lowered [12]. For the $\beta_{0} \rightarrow \beta$ reaction only a small shift to lower temperatures was observed [13].

Comparison of the experimentally determined phase evolution of alloy A with the phase diagrams shown in Fig. 1 reveals that the eutectoid temperature of alloy A determined by HEXRD is significantly higher than that suggested by the section at $43 \% \mathrm{Al}$, but only slightly higher than that in the section for $45 \% \mathrm{Al}$. The experimentally determined $T_{\alpha}$ lies higher than $T_{\alpha}$ of the section for $43 \% \mathrm{Al}$ but significantly below that for $45 \% \mathrm{Al}$. However, all phase fields occurring in the phase diagrams were also found in the HEXRD experiment. Alloy B exhibits large $\alpha$-phase fractions only above $1200^{\circ} \mathrm{C}$. The small volume fractions present at lower temperatures can be indicative of small remnant $\alpha$-phase fractions introduced during the production process. Another possibility is that the boundary between the $\left(\alpha_{2}+\beta+\gamma\right)$ and the $(\beta+\gamma)$ phase regions is close to the composition of alloy B. From the course of the $\alpha_{2} / \alpha$-phase fraction it is tempting to speculate that $\left(\alpha_{2}+\beta+\gamma\right)$ is stable at room temperature and the $(\beta+\gamma)$ phase region is entered at elevated temperatures only. $T_{\alpha}$ of alloy B lies in between the $\alpha$-transus temperatures suggested by the two sections of the ternary phase diagrams shown in Fig. 1. The role of the specimens Al-content has to be regarded when comparing the experimental data with the phase diagrams. It is well known, that in TiAl alloys $T_{\alpha}$ is strongly dependent on the $\mathrm{Al}$ content [4]. Both alloys have Al contents well below the nominal 45\%. With the exception of the eutectoid temperature for alloy A, however, all experimentally determined values lie in between the temperatures suggested by the two sections shown in Fig. 1.

Evaluation of phase evolution, transition and ordering temperatures by in-situ HEXRD experiments suffers from certain limitations. One of the drawbacks is that due to the non-zero heating rate, the transition temperatures are shifted to higher values. It has been shown that the $\alpha_{2} \rightarrow \alpha$ and the $\beta_{0} \rightarrow \beta$ disordering temperatures are rather insensitive to heating rate, whereas $T_{\alpha}$ 
shows a pronounced dependency [12]. However, the ambiguities introduced by this effect are significantly smaller than the differences between the HEXRD data and the phase diagrams shown in Fig. 1. Additionally, at high temperatures, $\alpha$ might be stabilized in surface-near regions due to the evaporation of $\mathrm{Al}$, which could lead to artefacts when evaluating phase fractions. This possibility was precluded by the examination of the specimen in the light-optical microscope after the experiment.

\section{Summary}

Sections through the ternary phase diagram Ti-Al-Mo at $43 \%$ and $45 \% \mathrm{Al}$ are compared to the phase evolutions of two nominal Ti-45Al alloys with additions of 3\% and 7\% Mo as determined by in-situ HEXRD. The transformation temperatures obtained for the investigated alloys lie in between the temperatures suggested by the two quasi-binary phase diagrams. The discrepancies are explained by the deviation in Al content of the investigated alloys from nominal composition. The HEXRD results allow a refinement of the Ti-Al-Mo phase diagram.

\section{Acknowledgements}

The support of the DESY management, User Office and beamline staff is highly acknowledged. Research activities leading to the presented results received funding from the European Community's Seventh Framework Programme (FP7/2007-2013) under grant agreement n 226716. A part of this study was conducted within the framework of the BMBF project O3X3530A.

\section{References}

[1] Structural Aluminides for Elevated Temperature Applications, edited by Y.-W. Kim, D. Morris, R. Yang and C. Leyens, Warrendale, TMS, (2008).

[2] Y.-W. Kim in. Structural Aluminides for Elevated Temperature Applications, edited by Y.-W. Kim, D. Morris, R. Yang and C. Leyens, Warrendale, TMS, p.215 (2008).

[3] R. Kainuma, Y. Fujita, H. Mitsui, I. Ohnuma and K. Ishida, Intermetallics 8, 855 (2000).

[4] H. Clemens, W. Wallgram, S. Kremmer, V. Güther, A. Otto and A. Bartels, Adv. Eng. Mat.10, 707 (2008).

[5] K.-D. Liss, A. Bartels, A. Schreyer, H. Clemens, Texture Microstruct 35, 219 (2003).

[6] Ge Czi-Min, E.N. Pylaeva, in Red Books Vol. IX (1963).

[7] T. Hamajima, G. Luetjering and S. Weissmann in Red Books, Vol. XVIII (1972).

[8] R. Hansen and R. Aravamudham, Metallkunde 61, 115 (1970).

[9] A.K. Singh and D. Banerjee, Metall. Mater. Trans. A 28, 1745 (1997).

[10] T. Lippmann, L. Lottermoser, F. Beckmann, R.V. Martins, T. Dose and R. Kirchhof, and A. Schreyer in: HASYLAB Annual Report, edited by W. Caliebe, W. Drube, K. Rickers. J.R. Schneider, HASYLAB/DESY, Hamburg (2007), p. 113.

[11] A.P. Hammersley, S.O. Svensson, M. Hanfland, A.N. Fitch, and D. Häusermann, High Pressure Res. 14, 235 (1996).

[12] T. Schmoelzer, K.-D. Liss, G.A. Zickler, I.J. Watson, L.M. Droessler, W. Wallgram, T. Buslaps, A. Studer and H. Clemens, submitted to Intermetallics (2010).

[13] I.J. Watson, K.-D. Liss, H. Clemens, W. Wallgram, T. Schmoelzer, T.Hansen and M. Reid, Adv. Eng. Mater. 11, 932 (2009). 